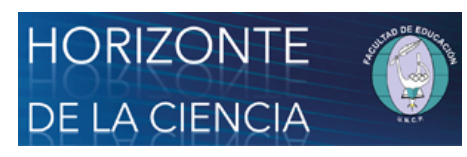

Horizonte de la Ciencia

ISSN: 2413-936X

horizontedelaciencia@uncp.edu.pe

Universidad Nacional del Centro del Perú

Perú

\title{
La estrategia de enseñanza y aprendizaje en la formación de profesionales de la Construcción Civil.
}

Ávila Rodríguez, Esmérido Evaristo; Vivanco Euvin, Andrea del Rosario; Rosado Hurtado, Alexandra Lily La estrategia de enseñanza y aprendizaje en la formación de profesionales de la Construcción Civil.

Horizonte de la Ciencia, vol. 10, núm. 18, 2020

Universidad Nacional del Centro del Perú, Perú

DOI: https://doi.org/10.26490/uncp.horizonteciencia.2020.18.409

Los autores otorgan el permiso a compartir y usar su trabajo manteniendo la autoría del mismo. Atribución no comercial (CC BY-NC) 4.0 


\title{
La estrategia de enseñanza y aprendizaje en la formación de profesionales de la
}

\section{Construcción Civil.}

\author{
The Teaching and Learning Strategy in the Training of Civil Construction Professionals \\ A estratégia de ensino e aprendizagem na formação de profissionais da Construção Civil \\ Yachachi yachakunap imanu lulayninkunata,yachanap ishpichi lulakuna allin yachakunap \\ ARETAKOPEROTANTYARORI YOMETANTSI EJATZITA YOTANETANTSI YOTAKOYETSIRORI \\ BETSIKIRORI PANKOTSIPE
}

Ora komantagantsi kantëgotiro ogotagantsi kara yogometaiganakeri antagetaigiro anchategapage

Esmérido Evaristo Avila Rodríguez Datos de los autores

DOI: https://doi.org/10.26490/

Universidad Técnica de Babahoyo, Cuba

uncp.horizonteciencia.2020.18.409

esmeridoavila@gmail.com

iD http://orcid.org/0000-0002-9332-1422

Andrea del Rosario Vivanco Euvin

Instituto Superior Técnico Eugenio Espejo, Ecuador

Recepción: 13 Marzo 2019

andrearv_07@hotmail.com

(D) http://orcid.org/0000-0002-1023-9811

Alexandra Lily Rosado Hurtado

Instituto Superior Técnico Eugenio Espejo, Ecuador

alirohu_63@hotmail.com

Recepción: 13 Marzo 2019

Aprobación: 01 Junio 2019

\section{Resumen:}

El desarrollo acelerado de la ciencia y la técnica permite que las personas tengan acceso a información actualizada sobre los procesos productivos y servicios que ejecutan los egresados de la carrera de Ingeniería Civil. Mediante la presente investigación se constató que en los currículos de la carrera no se tiene en cuenta el desarrollo de habilidades en el uso y manejo de las normas de calidad. Los docentes que imparten las materias técnicas en dicha especialidad pueden aplicar ejercicios tendientes al desarrollo en los educandos de dichas habilidades. Se propone un sistema de acciones que permitan al docente desarrollar habilidades en sus educandos y para que los educandos se apropien de dichas habilidades. Estos ejercicios se estructuran a partir de las estrategias de enseñanza y de aprendizaje, partiendo del desarrollo de habilidades en el uso de las normas de calidad.

Palabras Clave: Habilidades, Estrategias de enseñanza, Estrategias de aprendizaje, Construcción Civil, Normas de calidad, Yachaykuna, imanu yachachina, imanu yachana, kawsanap ishpichi, allikap tupuynin, ashimitsatsimoterine, aretakoperotantyarori yometantsi, aretakoperotantyarori yotanetantsi, betsikirori pankotsipe, aretakotantyarori saikotantsi iroperotantsi, okantagani kara pogomaeantaganiri, okantagani kara, patotagantsi irasi antaigiro pongotsipage.

\section{NotAS DE AUTOR}

Datos Esmérido Evaristo Ávila Rodríguez. Investigador y Profesor Asistente de Derecho, Ciencias Sociales, Pedagogía, Filosofía, Literatura y Artes. Máster de los en Cultura Latinoamericana por la Universidad de las Artes, de La Habana, Cuba. autores

Andrea del Rosario Vivanco Euvin. Docente de Ciencias de la Educación.

Alexandra Lily Rosado Hurtado. Docente de Ciencias de la Educación.

esmeridoavila@gmail.com 


\begin{abstract}
:
The accelerated development of science and technology allows people to have access to up-to-date information on the production processes and services performed by graduates of the Civil Engineering career. Through the present investigation, it was found that the development of skills in the use and management of quality standards is not taken into account in the curricula of the career. The teachers who teach the technical subjects in this specialty can apply exercises tending to the development in the learners of these skills. This research proposed a system of actions that allow teachers to develop the skills in their students so that students use them metacognitively. These exercises are structured from teaching and learning strategies, starting from the development of skills in the use of quality standards.
\end{abstract}

KEYWORDS: Skills, Teaching Strategies, Learning Strategies, Civil Construction, quality standards.

\title{
Resumo:
}

O desenvolvimento acelerado da ciência e da tecnologia permite que as pessoas tenham acesso a informações atualizadas sobre os processos produtivos e serviços que executam os egressos do Curso de Engenharia Civil. Através dessa pesquisa constatouse que nos currículos do Curso não se tem em conta o desenvolvimento de habilidades no uso e gerenciamento das normas de qualidade. Os docentes que ministram as disciplinas técnicas em dita especialidade poderiam aplicar exercícios voltados para o desenvolvimento dos estudantes de ditas habilidades. Propõe-se um sistema de ações que permitam ao docente desenvolver habilidades em seus educandos e que os alunos se apropriem das mesmas. Esses exercícios estruturam-se a partir das estratégias de ensino e da aprendizagem baseados no desenvolvimento de habilidades no uso das normas de qualidade.

Palavras-chave: Habilidades, estratégias de ensino, estratégias de aprendizagem, construção civil, padrões de qualidade.

\section{INTRODUCCIÓN}

Existe una falencia en el desarrollo de habilidades en el campo de la aplicación de las normas de calidad. Por ello este trabajo está dirigido a mostrar posibles modelos pedagógicos que permitan a los futuros egresados de la carrera de Ingeniería Civil adquirirlas. Se parte de la conceptualización del término habilidades, como elemento del pensamiento y como vínculo entre objeto y sujeto.

Según el Diccionario de la Real Academia de la Lengua Española: "La resultante de una combinación de características de ingeniería y fabricación, determinante del grado de satisfacción que el producto proporcione al consumidor, durante su uso". (Real Academia de la Lengua Española, 2017). Por tanto, la calidad en el campo de la ingeniería civil tiene que ver con el proceso ingenieril y de fabricación de modelos para la construcción y de sus servicios asociados.

Para desarrollar habilidades que permitan su manejo adecuado por parte de los profesionales egresados de dicha carrera se debe recurrir a las estrategias de enseñanza y de aprendizaje, que le permitan al estudiante evaluar y mejorar los estándares de calidad de sus productos y servicios.

Los docentes tienen que hacer uso de las estrategias que permitan desarrollar habilidades en sus estudiantes. Para la investigación presente se tuvo en cuenta el perfil del egresado de cuatro universidades ecuatorianas y se consultó el de universidades de Colombia, México y Venezuela. Como resultado, se llegó a la conclusión de que en Ecuador no se tiene en cuenta, en los currículos de las principales universidades, el desarrollo de habilidades para evaluar y aplicar las normas de calidad por parte de sus egresados.

\section{DesarRollo}

El egresado de la carrera de Ingeniería Civil debe poseer las habilidades necesarias para el eficiente desarrollo de su actividad laboral. Por ello el currículo debe contemplar actividades que propicien la adquisición de las mismas. En la mayoría de las universidades e institutos donde se imparte esta especialidad se coincide, generalmente, en el perfil del egresado. Tomamos al azar uno de ellos, correspondiente a la Escuela Politécnica del Litoral de Ecuador, un centro de altos estudios con una sólida experiencia en las diferentes ingenierías:

Perfil de Egreso: 
Los graduados tendrán habilidades para realizar estudios definitivos en su campo de acción; diseñar y ejecutar obras civiles en las especializaciones de hidráulica, estructuras, construcción, carreteras, sanitaria y ambiental, geotecnia y suelos; desarrollar proyectos de remodelación de edificios; proyectos de edificaciones, puentes, pasos a desnivel, muelles, túneles, obras viales, obras hidráulicas, obras sanitarias, obras geotécnicas, medio ambiente, etc., así como emprender negocios relacionados con la profesión. (Espol, 2017)

De la misma Universidad se muestra el Perfil Educacional:

El Ingeniero Civil puede desempeñarse como gerente de una empresa pública o privada; ser consultor, constructor, fiscalizador de obras civiles; dirigir proyectos ejercer profesionalmente en las especializaciones de hidráulica, estructuras, construcción, carreteras, sanitaria y ambiental, geotecnia y suelos; desempeñarse como asistente y residente de obra en Compañías Constructoras Privadas e Instituciones Públicas, así como en empresas de consultoría en obras civiles. (Espol, 2017)

Como se observa en el párrafo anterior, el objetivo formativo es el desarrollo de habilidades, que le permita al futuro egresado, con un amplio perfil, desempeñarse en diversos campos de la ingeniería de la construcción de obras civiles.

El concepto de habilidades ha sido ampliamente abordado por investigadores de diversas ramas del saber, entre las que se cuentan la Psicología, la Sociología y en especial la Pedagogía.

Para Álvarez, al analizar las características del currículo, las habilidades son: "estructuras del pensamiento, lógicas, motoras, que permiten asimilar, utilizar y exponer los conocimientos. Se estructuran en operaciones que constituyen su conformación técnica. Se forman y desarrollan a través de la ejercitación de las acciones y se convierten en modos de actuación que dan solución a tareas teóricas y prácticas". (Álvarez, 1997). Se pudiera resumir, entonces, que las habilidades, para este autor, son esas estructuras mentales que se logran mediante la sistematización de ejercicios y que luego se convierten en modos de actuación automáticos, con mayor o menor grado de independencia.

Por su parte, para el profesor de la Universidad de Oriente, H. Fuentes, las habilidades son: "el modo de interacción del sujeto con los objetos, que se dan en la actividad y la comunicación, constituyen el contenido de las acciones que el sujeto realiza con un objetivo, juegan un papel importante en la autorregulación del sujeto y en el conocimiento de sí mismo" (Fuentes, 1999). Por lo que a diferencia del autor anteriormente citado, éste ve el desarrollo de las habilidades desde la óptica de su interrelación social, donde el lenguaje tiene un papel preponderante. En este proceso el sujeto se conoce y regula sin necesidad de un apoyo externo, necesario en etapas donde no están desarrolladas las habilidades.

Existe una reciprocidad entre el proceso de aprendizaje y la adquisición de habilidades. Mediante el aprendizaje de nuevos conocimientos el sujeto va paulatinamente apropiándose de habilidades para la realización de determinadas acciones. Mientras que en la medida en que este propio sujeto desarrolla sus habilidades, está asistiendo a una asimilación de saberes. Esta dualidad se manifiesta en el propio sistema de relaciones sociales. La solución de problemas obliga al sujeto a desarrollar habilidades.

La Doctora Azaharez Fernández y la Máster Rodríguez Suárez consideran que las habilidades se utilizan para:

- Caracterizar los rasgos esenciales que se observan.

- Precisar las condiciones en que ocurre.

- Conocer cómo está hecho un objeto.

- Establecer su esencia y el mecanismo de su desarrollo.

- Utilizar ejemplos prácticos.

- Lograr que el estudiante pueda denominar, definir, interpretar, comparar, clasificar, medir, (en caso que se trate de magnitudes).

- Precisar las unidades que componen el conjunto. (Rodríguez Suárez \& Azaharez Fernández, 2017)

El sistema operacional que compone una acción en la que interactúan el sujeto y el objeto, persiguen un fin que se asume en el proceso realizado para llegar a un determinado punto de desarrollo. Por lo tanto la 
habilidad estará dada en dicho proceso. Álvarez hace énfasis en la sistematización de las habilidades que se trabajan o se quieren lograr durante el currículum correspondiente a la habilitación del futuro profesional. Para este pedagogo el profesional debe llegar a adquirir una habilidad máxima que pueda:

- Aplicar conocimientos.

- Actuar y transformar su objeto de trabajo.

- Resolver, (y esto es muy importante para el autor), los problemas generales y frecuentes que enfrentará en sus ámbitos de actuación. (Álvarez I. , 1999)

Las habilidades constituyen el elemento esencial a lograr en la formación de un profesional en cualquier rama del saber humano. En ellas se fundamentan las acciones básicas de la teoría y la praxis de los que debe apropiarse el sujeto. Para transformar el objeto, el sujeto debe contar con habilidades básicas y específicas que le permitan accionar sobre sus propiedades y modificarlas en base a las necesidades que se presenten en el espacio y ámbito temporal de su actuación.

El profesional contemporáneo debe tener dominio de las habilidades para resolver los complejos problemas que se presentan. Para ello la educación se basa en los cuatro pilares fundamentales (saber, saber hacer, ser y convivir).

La carrera de Ingeniería Civil debe formar graduados con sólidos saberes, habilidades y ética que los habilite para poder desarrollar el trabajo en la era de las nuevas tecnologías de la informática y las comunicaciones. Del dibujo manual se pasó a la elaboración, mediante programas informáticos, de planos y simulaciones de objetos conocidos, rediseñados o innovados; nuevos instrumentos de medición y cálculo, generalmente digitalizados; la optimización del tiempo con el uso de calculadores y computadoras y hasta desde los teléfonos celulares, permiten al futuro profesional la adquisición de nuevas habilidades. Por ello los docentes debemos elaborar currículos, preparar clases y organizar el proceso docente educativo teniendo en cuenta estas nuevas tecnologías y los nuevos avances de la ciencia.

El profesional en Ingeniería Civil egresa con la capacidad de análisis y redimensionamiento de edificios, empleando programas informáticos profesionales; como tal formula y estima el coste de proyectos, supervisa y revisa y realiza informes; identifica y analiza los problemas tecnológicos de su radio de acción constructivo, con el fin de garantizar los patrones de calidad y seguridad normados; proyecta y calcula obras hidráulicas; prevé y acciona sobre los problemas del ambiente vinculados con una edificación; reconoce particularidades sociales de su zona de trabajo y realiza investigaciones para la búsqueda de soluciones adecuadas.

También el profesional egresado de la Ingeniería Civil está dotado de habilidades que le permiten proponer innovaciones para el desarrollo industrial y urbano, el uso eficiente de los recursos hídricos, la protección ecológica, vinculada a la salud pública y proyectos viales, todos encaminados al mejoramiento de la vida y el logro del estado de bienestar, adecuando la relación del hombre y el medio donde se desenvuelve su actividad.

En estas habilidades mencionadas se resume la actuación profesional del egresado de la carrera de Ingeniería Civil.

En ésta, como en otras carreras es muy importante la aplicación de las normas de calidad. Por ello dentro del currículo se debe desarrollar habilidades para el empleo de las mismas en la elaboración, análisis y evaluación de los proyectos.

La normalización y control de la calidad no forma parte del currículum de la carrera de Ingeniería Civil de las universidades ecuatorianas y de muchas universidades e institutos tecnológicos del Ecuador y el mundo. Siendo tan importante esta actividad y el desarrollo de habilidades en la misma para mejorar la calidad de las obras civiles, es significativo que no se tenga en cuenta. Los profesionales egresan con falencias en estas habilidades de la evaluación de la calidad.

Según Arredondo:

Una norma de calidad es un documento, establecido por consenso y aprobado por un organismo reconocido (nacional o internacional), que proporciona para un uso común y repetido, una serie de reglas, directrices o características para las 
actividades de calidad o sus resultados, con el fin de conseguir un grado óptimo de orden en el contexto de la calidad. (Arredondo, 2017)

Entre las normas de calidad la más utilizada a nivel internacional es la ISO, que se le denomina así por sus siglas en inglés, International Organization for Standardization, (Organización Internacional de Estandarización), sistema de normalización internacional para productos y servicios de diferentes áreas.

Estas normas sirven como patrón para los estándares de calidad de productos y servicio. Por ello, en la construcción de obras es aplicable. Si se tiene en cuenta que la calidad es primordial en el control de las edificaciones, entonces se hace necesario desarrollarlas en los profesionales de estas materias y evaluar su nivel de aprendizaje.

El desarrollo en los estudiantes de habilidades para evaluar la calidad de los procesos y resultados de su gestión profesional en el campo de la ingeniería civil debe ser un objetivo primordial del proceso de enseñanza / aprendizaje en las universidades especializadas en esta carrera.

Para lograr este objetivo se hace necesario que el docente domine el entorno en que se encuentra la ciencia y la tecnología actuales y las estrategias de enseñanza y aprendizaje.

El mundo asiste a un acelerado desarrollo de las ciencias y las tecnologías. El caudal de conocimientos aumenta exponencialmente en todas las ramas del saber humano. La mecánica cuántica, las computadoras y la biología molecular, vinculadas a las altas tecnologías, como la biotecnología, las ciencias informáticas, la comunicación, las ciencias espaciales, la nanotecnología, la mecatrónica, la robótica, crean nuevos productos, sin los cuales es imposible imaginar la existencia del hombre. Y, aunque hay un debate ético a su alrededor, se considera que significan el motor impulsor del desarrollo humano.

Aplicar creativamente el conocimiento de la ciencia y la técnica, mediante el vehículo de la ingeniería, conduce al desarrollo y progreso siempre que los profesionales que los utilicen cuenten con las habilidades necesarias para su implementación en la praxis. Se asiste a una revolución productiva en que los actores cuentan con nuevas herramientas, dinámicas y complejas en su manejo, pero que permiten el avance económico y social hacia un mejor nivel de vida. El ingeniero civil debe resolver adecuaciones constructivas que se correspondan con las nuevas realidades tecnológicas y que empleen los nuevos productos resultantes de las modernas tecnologías. En este punto se funden dos elementos importantes: las habilidades para su ejecución y la medición de la calidad de lo proyectado y ejecutado.

No basta con un profesional experto en su área de trabajo, sino que el nuevo ingeniero debe contar con una preparación técnica y humanística, esencial en la nueva era globalizada.

Fernando Romo, Director de Ingeniería Civil de la Universidad San Francisco de Quito, en: "La posesión y uso del conocimiento como base del desarrollo económico", expone que:

En este marco de referencia, el nuevo ingeniero del siglo XXI debe ser un profesional de excelentes niveles de preparación profesional en su área de especialización, con sólida formación humanística en artes liberales, buscador incansable e insaciable de nuevos y actualizados conocimientos científicos y tecnológicos, que puedan ser utilizados para encontrar soluciones prácticas y eficientes a las cada vez más exigentes necesidades de nuestra sociedad y para satisfacer los sueños, cada vez más ambiciosos del hombre moderno, en todos los campos del conocimiento. (Universidad San Francisco de Quito, 2017)

Empero en ese proceso es necesario la formación de habilidades en el conocer y el ejecutar. Para ello se debe tener en cuenta las estrategias de enseñanza y las estrategias de aprendizaje, dos conceptos interrelacionados en el proceso docente educativo.

\section{Estrategias DE ENSEÑANZA}

Estas se pueden conceptualizar como las ayudas que presenta el docente para que el estudiante realiza una profundización en el contenido de la información que se le proporciona. Constituye la correspondencia entre los métodos usados por el docente y que están encaminados al logro de aprendizajes significativos. Para ese fin 
se debe realizar un adecuado diseño, establecer un correcto programa, elaborar y realizar los contenidos que se deben aprender por el estudiante, tanto de manera oral como escrita. O lo que es lo mismo, una adecuada planeación del proceso de la clase. Para ello el docente debe tener en cuenta las características de sus educandos individualmente, así como del grupo que conforman.

Celia Trujillo Martínez, recomienda:

Tanto las características del grupo como el contenido de la materia están íntimamente relacionados con la forma de aprender de los mismos, también es importante contemplar los aspectos individuales, algunos alumnos son visuales, otros auditivos y unos más kinestésicos, por lo tanto, lo más recomendable es utilizar diferentes estrategias donde se involucren todos los elementos para que cada uno tenga la fuente de información acorde a su necesidad, ya que si nos abocamos a uno solo, alguno quedará confundido. (Martínez Trujillo, 2017).

De esta manera, si lo relacionamos con el interés del docente de lograr en el estudiante habilidades, entonces sería necesario diseñar una estrategia formativa en el área deseada. En el caso de las habilidades para aplicar en la ingeniería civil las normas de calidad se debe, primero enseñar su contenido y alcance y luego su aplicación y evaluación.

En el currículo se dicará tiempo a clases, tanto teóricas como prácticas, para su manejo. En ellas se debe desarrollar la observación, el análisis, la formulación de hipótesis y la búsqueda de soluciones, desde la construcción del conocimiento por el propio estudiante, o sea, el logro de una adecuada autonomía, sin recurrir a la memorización, contraria al aprendizaje significativo. (Díaz Barriga, 2010). Este aprendizaje conlleva el fomento del pensar crítico y el manejo provechoso y sintetizado de la información.

\section{EsTrategias DE APRENDIZAJE}

En cuanto a las estrategias de aprendizaje, el sujeto activo, que es el estudiante, debe imponerse objetivos, retos y fines, que le permitan avanzar y autoevaluarse. Realiza acciones para apropiarse del conocimiento, interiorizar la información que recibe y lograr un aprendizaje significativo.

Según Guillermo Michel, el estudiante debe autoevaluarse, a través de preguntas como:

“¿Qué pretendo?, ¿Qué quiero aprender?, ¿Para qué?, ¿Por qué?, ¿Qué formas de actuar, de pensar y de sentir voy a obtener como resultado de mis experiencias?, ¿A dónde quiero llegar? ¿Cómo voy a lograr lo que quiero?, ¿Cuándo?, ¿En qué momento?” (Michel, 2008). Estas se hacen con el objetivo de precisar los fines y definir el camino a seguir en el proceso de adquisición de habilidades. Cuando se transita por el camino del aprendizaje significativo el estudiante se apropia del contenido y lo transforma creativamente. Las características del proceso de aprendizaje varían según las características del estudiante. A esto se le llama estilo de aprendizaje.

Estrategias utilizadas por el estudiante y que las adecua a su estilo de aprendizaje son:

a) Lectura y comprensión

b) Análisis y síntesis

c) Recursos gráficos

El estudiante emplea las estrategias con la capacidad de analizar lo conocido mediante la reflexión, el análisis y la síntesis de lo aprehendido.

En este contexto desarrollar las habilidades relativas al uso y manejo de las normas de calidad en la carrera de Ingeniería Civil es vital y necesaria, ya que no se concibe el presente sin la normalización; y un egresado de ingeniería que tiene la responsabilidad social de general productos y servicios con calidad, dado su impacto en áreas complejas de la vida humana y la salud planetaria. 


\section{Conclusiones}

Las habilidades constituyen construcciones sistémicas de concientización de acciones reiteradas que se aprehenden en el estudiante y son producto de la interacción entre el sujeto y el objeto. En los currículos de la carrera de Ingeniería Civil no existe un tiempo diseñado para la adquisición de habilidades en el trabajo con las normas de calidad. Esto dificulta el desempeño del egresado. Para desarrollar las habilidades en este campo, se hace necesario aplicar las estrategias de enseñanza y de aprendizaje, como hábitos de estudio. Estas estrategias se formalizan en la interacción con el medio circundante de los educandos. Se hace necesario condicionar el las facultades correspondientes el estudio de lo referido a la calidad para un mejor desempeño del profesional del siglo XXI.

\section{REFERENCIAS}

Álvarez, C. (1997). Hacia un currículum integral y contextualizado. La Habana: Academia.

Álvarez, I. (1999). El proceso y sus movimientos. Modelo de la dinámicadel proceso docente educativo en la educación superior. Santiago de Cuba: Universidad de Oriente.

Arredondo, J. (15 de octubre de 2017). www.monografias.com/trabajos91/las-normas-iso/las-normasiso.shtml\#ixzz4wx4I13Z1.

Díaz Barriga, F. (2010). Estrategias docentes para un aprendizaje significativo. México : Editorial McGraw-Hill.

Espol. (14 de octubre de 2017). www.espol.edu.ec/es/facultades/FICT/carrera/ingenieria-civil. Obtenido de http:// www.espol.edu.ec/es/facultades/FICT/carrera/ingenieria-civil

Fuentes, H. (1999). Dinámica del proceso docente educativo en la educación superior. Monografía CEES "Manuel Franciasco Gran". Santiago de Cuba: Universidad de Oriente.

Martínez , C. (14 de octubre de 2017). http://www.monografias.com/trabajos98/sobre-estrategias-de-ensenanzaaprendizaje/sobre-estrategias-de-ensenanza-aprendizaje.shtml\#ixzz4wrEzOc9w.

Michel, G. (2008). Aprende a aprender. México: Editorial Trillas.

Rodríguez, A., \& Azaharez, O. T. (2017). Reingeniería como metodología de enseñanza para la formación de habilidades del ingeniero mecánico. Magazine de las Ciencias, 51-60.

Universidad San Francisco de Quito. (14 de octubre de 2017). http://www.usfq.edu.ec/programas_academicos/ colegios/politecnico/carreras/Paginas/ingenieria_civil.aspx.

Los autores otorgan el permiso a compartir y usar su trabajo manteniendo la autoría del mismo.

CC BY-NC 